\title{
Microcirculation and its relation to continuous subcutaneous glucose sensor accuracy in cardiac surgery patients in the intensive care unit
}

\author{
Sarah E. Siegelaar, MD, PhD, ${ }^{\mathrm{a}}$ Temo Barwari, MSc, ${ }^{\mathrm{a}}$ Jeroen Hermanides, $\mathrm{MD}, \mathrm{PhD},{ }^{\mathrm{a}}$ \\ Peter H. J. van der Voort, MD, PhD, ${ }^{b}$ Joost B. L. Hoekstra, MD, PhD, ${ }^{a}$ and J. Hans DeVries, MD, PhD ${ }^{\mathrm{a}}$
}

\begin{abstract}
Objective: Continuous glucose monitoring could be helpful for glucose regulation in critically ill patients; however, its accuracy is uncertain and might be influenced by microcirculation. We investigated the microcirculation and its relation to the accuracy of 2 continuous glucose monitoring devices in patients after cardiac surgery.
\end{abstract}

\begin{abstract}
Methods: The present prospective, observational study included 60 patients admitted for cardiac surgery. Two continuous glucose monitoring devices (Guardian Real-Time and FreeStyle Navigator) were placed before surgery. The relative absolute deviation between continuous glucose monitoring and the arterial reference glucose was calculated to assess the accuracy. Microcirculation was measured using the microvascular flow index, perfused vessel density, and proportion of perfused vessels using sublingual sidestream dark-field imaging, and tissue oxygenation using near-infrared spectroscopy. The associations were assessed using a linear mixed-effects model for repeated measures.
\end{abstract}

Results: The median relative absolute deviation of the Navigator was $11 \%$ (interquartile range, $8 \%-16 \%$ ) and of the Guardian was $14 \%$ (interquartile range, $11 \%-18 \% ; P=.05$ ). Tissue oxygenation significantly increased during the intensive care unit admission (maximum 91.2\% [3.9] after 6 hours) and decreased thereafter, stabilizing after 20 hours. A decrease in perfused vessel density accompanied the increase in tissue oxygenation. Microcirculatory variables were not associated with sensor accuracy. A lower peripheral temperature (Navigator, $\mathrm{b}=-0.008, P=.003$; Guardian, $\mathrm{b}=-0.006, P=.048$ ), and for the Navigator, also a higher Acute Physiology and Chronic Health Evaluation IV predicted mortality $(b=0.017, P<.001)$ and age $(b=0.002$, $P=.037)$ were associated with decreased sensor accuracy.

Conclusions: The results of the present study have shown acceptable accuracy for both sensors in patients after cardiac surgery. The microcirculation was impaired to a limited extent compared with that in patients with sepsis and healthy controls. This impairment was not related to sensor accuracy but the peripheral temperature for both sensors and patient age and Acute Physiology and Chronic Health Evaluation IV predicted mortality for the Navigator were. (J Thorac Cardiovasc Surg 2013;146:1283-9)

Intensive glucose control is widely practiced in the intensive care unit (ICU). However, the associated frequent glucose measurements are time consuming, and no information is available about the glucose values between those measurements. The occurrence of hypoglycemia has been independently associated with an increased risk of

From the Department of Internal Medicine, ${ }^{\text {a }}$ Academic Medical Centre, Amsterdam, The Netherlands; and Department of Intensive Care Medicine, ${ }^{\mathrm{b}}$ Onze Lieve Vrouwe Gasthuis, Amsterdam, The Netherlands.

This study was supported by a European Foundation for the Study of Diabetes/LifeScan research grant.

Disclosures: J. H. DeVries or institutions to which he is connected received research support and fees from speaking engagements from Abbott Diabetes Care and Medtronic and is on an advisory board of Abbott Diabetes Care. The other authors have nothing to disclose with regard to commercial support

Received for publication Nov 28, 2012; revisions received May 6, 2013; accepted for publication June 14, 2013; available ahead of print July 22, 2013

Address for reprints: Sarah E. Siegelaar, MD, PhD, Department of Internal Medicine, Academic Medical Center, Rm F4-257, Meibergdreef 9, Amsterdam, 1105 AZ, The Netherlands (E-mail: s.e.siegelaar@amc.uva.nl).

$0022-5223 / \$ 36.00$

Copyright () 2013 by The American Association for Thoracic Surgery

http://dx.doi.org/10.1016/j.jtcvs.2013.06.017 respiratory complications and prolonged hospital and ICU lengths of stay after cardiac surgery. ${ }^{1}$ Continuous glucose monitoring (CGM) could be a step forward by decreasing severe hypoglycemia frequency ${ }^{2}$ and increasing the time within the blood glucose target range. Although we recently reported promising results for continuous glucose sensor accuracy in cardiac surgery patients, ${ }^{3}$ a patient population that seems to benefit from intensive insulin treatment, ${ }^{4}$ other studies have reported suboptimal accuracy with the commercially available systems. ${ }^{5,6}$

A part of the accuracy problem in critically ill patients could have been because the current commercially available needle-type continuous glucose sensors measure the glucose concentrations in the interstitial fluid and not directly in the blood. The transport of molecules such as glucose to the interstitial fluid is dependent on the glucose supply to the tissue and, therefore, on microcirculatory function, although the exact interstitial glucose kinetics in response to vascular changes are unknown. In critically ill patients, the microcirculatory function has been altered, ${ }^{7,8}$ which might negatively affect sensor performance. 


$\begin{array}{ll}\text { Abbreviations and Acronyms } \\ \text { APACHE } & =\text { Acute Physiology and Chronic Health } \\ \text { IV PM } & \text { Evaluation IV predicted mortality } \\ \text { CGM } & =\text { continuous glucose monitoring } \\ \mathrm{HI} & =\text { heterogeneity index } \\ \mathrm{ICU} & =\text { intensive care unit } \\ \mathrm{IQR} & =\text { interquartile range } \\ \mathrm{MFI} & =\text { microvascular flow index } \\ \mathrm{PVD} & =\text { perfused vessel density } \\ \mathrm{PPV} & =\text { proportion of perfused vessels } \\ \mathrm{RAD} & =\text { relative absolute deviation } \\ \mathrm{SDF} & =\text { sidestream dark-field } \\ \mathrm{StO} & =\text { tissue oxygenation }\end{array}$

In the present investigator-initiated study, we investigated the microcirculation in patients after cardiac surgery in the ICU and assessed whether microcirculatory variables and other patient-related factors were associated with the accuracy of the Guardian Real-Time (Medtronic Minimed, Northridge, Calif) and FreeStyle Navigator (Abbott Diabetes Care, Alameda, Calif) continuous glucose monitoring systems.

\section{METHODS \\ Patients}

We performed a prospective observational study in a 20-bed mixed ICU in the Onze Lieve Vrouwe Gasthuis (Amsterdam, The Netherlands). The institutional review board approved the study. We included patients aged $>18$ years who were to undergo elective cardiac surgery: coronary artery bypass grafting and/or valve surgery. We excluded patients with an abdominal condition that would impair sensor insertion. The eligible patients received an information letter $\geq 1$ week before admission and were asked to give written informed consent after additional explanation of the study the day before surgery.

\section{Glucose Monitoring}

We used 2 needle-type sensors (Guardian Real-Time, Medtronic Minimed, and FreeStyle Navigator, Abbott Diabetes Care). These sensors quantify the interstitial glucose concentrations by way of the glucose oxidation reaction, with the enzyme immobilized on a subcutaneously inserted electrode. After we obtained informed consent, the sensors were inserted in the abdominal wall on either side of the umbilicus the day before surgery and were calibrated to allow stabilization of the signal. On arrival at the ICU after surgery, the device's internal clock was synchronized with the bedside computer, and both devices were calibrated simultaneously. Additional calibrations were performed according to the manufacturers' instructions. The devices were removed after 48 hours of ICU admission or earlier, when the patient was discharged.

A reference arterial blood glucose value was measured using the AccuChek handheld glucose measurement device (Performa II, lot 320098; Roche/Hitachi, Basel, Switzerland) every 2 hours. These samples were used as calibration when needed and as reference otherwise. An in-house quality assurance study showed that the slope of the regression between this point-of-care measurement method and arterial glucose measurement by blood gas analysis was 1.0 (95\% confidence interval $1.00-1.02, \mathrm{n}=1393$, sample range 2.9-30.0 mmol/L; Passing-Bablok regression), and $95 \%$ of the absolute differences between the reference and point-of-care measurements were lower than $15 \%$, meeting the International Organization for Standardization protocol number 15197 guideline. The results were stored in the ICU's clinical information system (iMDSoft; MetaVision, Tel Aviv, Israel). Using a dynamic computerized algorithm implemented in $2001,{ }^{9}$ glucose values of 5.0 to $8.0 \mathrm{mmol} / \mathrm{L}$ were targeted. The nursing staff adjusted the insulin infusion rate according to the current arterial glucose value and the rate of glucose change determined from the previous 5 measurements and did not act on the sensor glucose values.

To assess the real-time accuracy of the devices, we calculated the relative absolute deviation (RAD) (|sensor value - reference glucose // reference glucose) between the reference glucose and sensor glucose values. For this purpose, we linked the reference value to the first available sensor value within 5 minutes after the reference value had been measured.

\section{Microcirculation Measurement}

We assessed the microcirculation sublingually, after removal of excess saliva, using a handheld sidestream dark-field (SDF) camera. This method has been previously described ${ }^{10}$ and is representative of the microcirculation of other vascular beds. ${ }^{11}$ Imaging was performed as soon as the patient arrived at the ICU, 2 and 4 hours thereafter, and at 8 AM, 12 PM, and 16 PM on the subsequent days until the patient was discharged from the ICU or at 48 hours after ICU admission, whichever came first. At each point, SDF recordings of $\geq 3$ different sublingual sites were recorded, stored, and scored in random order to prevent bias. The recordings were excluded when no flow was visible in the large vessels, indicating a pressure artifact, the presence of excess saliva making it impossible to reliably visualize all vessels, and/or excess movement. We analyzed vessels with a diameter of $<20 \mu \mathrm{m}$, in line with previous data, ${ }^{12}$ because these capillaries and small venules are considered to be the most important in nutrient (ie, glucose) transport. Together with every SDF recording, a reference plasma glucose measurement was obtained to calculate sensor accuracy.

We scored the microvascular flow index (MFI), proportion of perfused vessels (PPV), and perfused vessel density (PVD) per sublingual site per patient. The 3 outcomes per measurement point per patient were then averaged according to the consensus statement on how to evaluate the microcirculation ${ }^{13}$ using the automated vascular analysis program, version 3.0 (Department of Medical Technological Development, Academic Medical Center, and MicroVision Medical, Amsterdam, The Netherlands). In brief, for the PVD and PPV, the vessel density was calculated as the number of vessels crossing 3 horizontal and 3 vertical equidistant lines divided by the total length of the lines. The perfusion of the crossing vessels was scored as follows: 0 , no flow; 1 , intermittent flow (flow present $<50 \%$ of the recording); 2 , sluggish flow (flow present $>50 \%$ but $<100 \%$ of the recording or continuous very slow flow); and 3, continuous flow. PVD was calculated as the number of crossing vessels with flow present (score 2 or 3 ) reported in $\mathrm{n} / \mathrm{mm}$. The PPV is the proportion of vessels with flow present (score 2 or 3 ). ${ }^{7}$ For the MFI, the predominant type of flow in 4 quadrants was determined according to the same scoring system. The MFI is the sum of these flow scores divided by the number of quadrants in which the vessel type is visible. ${ }^{14}$ The heterogeneity index (HI) per measurement point for the PVD was calculated by dividing the difference between the lowest and highest value by the mean to objectify the intrasite differences per measurement point. ${ }^{15}$

The MFI was scored by 2 of us (S.E.S. and T.B.) and was compared and revised by consensus when the separate scores differed by $>1$ point. The final score was obtained by averaging the separate scores. The separate scores never differed $>1$ point. The mean intraobserver difference was 0.06 points (range, $0.00-0.31$ ) on the 3 -point MFI scale. The points did not differ at all between the 2 investigators $40 \%$ of the time. The PPV and PVD were scored by 1 of us (S.E.S.), and the intraobserver variability was determined by reanalyzing 17 randomly chosen movie sequences after 4 weeks. The intraclass correlation coefficient was calculated using a 2-way random model to determine the absolute agreement. The intraclass correlation coefficient for PVD was 0.94 ( $95 \%$ confidence interval, 
0.84-0.98) and for PPV was 0.94 (95\% confidence interval, 0.84-0.98), indicating good intraobserver agreement.

The tissue oxygenation $\left(\mathrm{StO}_{2}\right)$ was measured using near-infrared spectroscopy to assess the microcirculatory function (InSpectra $\mathrm{StO}_{2}$ Tissue Oxygenation Monitor; Hutchinson Technology, Hutchinson, Minn). The $\mathrm{StO}_{2}$ is a measure of oxygen consumption of the tissue and a predictor of organ dysfunction. ${ }^{16}$ It reflects the ratio of oxygenated hemoglobin to total hemoglobin in the microcirculation measured by the absorption of near infrared light (wavelength, 700-1000 nm). The probe was placed on the thenar muscle at ICU arrival and removed after 48 hours or on discharge from the ICU.

\section{Other Variables}

Other factors possibly influencing CGM accuracy were also obtained at every reference glucose measurement: mean arterial pressure, peripheral skin temperature, rectal temperature, pulse oximeter oxygen saturation, and dosing of vasoactive medication. The Acute Physiology and Chronic Health Evaluation IV predicted mortality (APACHE IV PM) was calculated for the first 24 hours of admission, and the Sequential Organ Failure score was obtained daily. Also, the European system for cardiac operative risk evaluation score, a method of calculating the predicted operative mortality risk of patients undergoing cardiac surgery, was recorded for every patient.

\section{Data Interpretation and Statistical Analysis}

The median and interquartile range (IQR) RAD per patient was calculated for each sensor, and both sensors were compared using a Wilcoxon signed ranks test for non-normally distributed paired data. For each sensor, all paired samples of the reference glucose values and matching next sensor values were plotted in a Clarke error grid, ${ }^{17}$ which is divided into 5 zones. Zones A and B represent values that are clinically acceptable. Zone A represents values that differed $\leq 20 \%$ from the reference value. Zone B represents values that differed $>20 \%$ but would not lead to a different treatment decision. Zone $\mathrm{C}$ represents values that would result in overcorrecting acceptable glucose values. Zone D represents values that are erroneously not corrected, and zone E represents values that would result in the opposite treatment (eg, glucose-lowering therapy in reaction to a hypoglycemic value). To describe the patient characteristics and overall circulatory function, the median and IQR or mean \pm standard deviation per patient were calculated (Tables 1 and 2).

The associations between sensor accuracy and individual circulatory variables were assessed using a linear mixed-effects model for repeated measures. Second, we built a multivariate model for each sensor that included those variables with a significant association with the accuracy of that specific sensor. Finally, the microcirculatory variables (MFI, $\mathrm{PVD}, \mathrm{PPV}$, and $\mathrm{StO}_{2}$ ) were forced in turn into the definite model to assess the possible independent effect on sensor accuracy. All analyses were performed using Predictive Analytics Software (PASW) statistics, version 18.0 (SPSS Inc, Chicago, Ill). $P<.05$ was considered statistically significant. The European Foundation for the Study of Diabetes/LifeScan did not have any role in the interpretation of the data.

\section{RESULTS}

We included 61 patients in the present study, of whom, 1 patient withdrew owing to cancellation of surgery because of febrile illness. Thus, 60 patients were available for the final analysis (48 men). The median age was 65 years (range, 25-85), and 16 patients had previously been diagnosed with type 2 diabetes. Most patients underwent only coronary artery bypass grafting $(\mathrm{n}=32)$. The median European system for cardiac operative risk evaluation score
TABLE 1. Patient characteristics $(n=60)$

\begin{tabular}{|c|c|}
\hline Characteristic & Value \\
\hline Male gender (n) & $48(80.0)$ \\
\hline Age (y) & $65.0(59.0-73.8)$ \\
\hline Diabetes (n) & $16(26.7)$ \\
\hline \multicolumn{2}{|l|}{ Procedure (n) } \\
\hline CABG & $32(53.3)$ \\
\hline Valve surgery & $16(26.7)$ \\
\hline CABG plus valve surgery & $12(20.0)$ \\
\hline APACHE IV PM & $0.01(0.003-0.02$ \\
\hline Maximal SOFA score & $6.0(5.3-7.0)$ \\
\hline EuroSCORE & $4.0(2.0-5.0)$ \\
\hline ICU stay $(\mathrm{h})$ & $23.0(19.0-45.8)$ \\
\hline ICU readmission $(\mathrm{n})$ & $6(10.0)$ \\
\hline Death in ICU or hospital (n) & 0 \\
\hline Glucose ICU & $8.2 \pm 2.1$ \\
\hline RAD Navigator (\%) & $11(8-15)$ \\
\hline RAD Guardian (\%) & $14(11-18)$ \\
\hline Dopamine $(\mu \mathrm{g} / \mathrm{kg} / \mathrm{min})(\mathrm{n}=60)$ & $1.62(1.03-2.34)$ \\
\hline Nitroglycerine $(\mu \mathrm{g} / \mathrm{kg} / \mathrm{min})(\mathrm{n}=57)$ & $0.22(0.16-0.34)$ \\
\hline Enoximon $(\mu \mathrm{g} / \mathrm{kg} / \mathrm{min})(\mathrm{n}=40)$ & $1.19(0.80-1.71)$ \\
\hline Ketanserine $(\mu \mathrm{g} / \mathrm{kg} / \mathrm{min})(\mathrm{n}=4)$ & $0.26(0.08-0.39)$ \\
\hline Noradrenaline $(\mu \mathrm{g} / \mathrm{kg} / \mathrm{min})(\mathrm{n}=1)$ & 0.09 \\
\hline MFI small vessels & $2.8(2.7-2.9)$ \\
\hline PPV small vessels & $0.97(0.96-0.99)$ \\
\hline PVD small vessels $(\mathrm{n} / \mathrm{mm})$ & $9.3(8.4-10.0)$ \\
\hline Heterogeneity index PVD & $0.17(0.13-0.21)$ \\
\hline $\mathrm{StO}_{2}(\%)$ & $90.3(87.1-92.0)$ \\
\hline $\mathrm{SpO}_{2}(\%)$ & $97.2(95.7-98.6)$ \\
\hline Rectal temperature $\left({ }^{\circ} \mathrm{C}\right)$ & $36.8(36.4-37.1)$ \\
\hline Peripheral temperature $\left({ }^{\circ} \mathrm{C}\right)$ & $32.8(31.6-33.6)$ \\
\hline $\mathrm{HF}$ (beats/min) & $84.2(74.4-90.0)$ \\
\hline MAP (mm Hg) & $73.6(69.3-81.0)$ \\
\hline Hemoglobin $(\mathrm{mmol} / \mathrm{L})$ & $5.8(5.6-6.4)$ \\
\hline Hematocrit $(\mathrm{L} / \mathrm{L})$ & $0.29(0.28-0.31)$ \\
\hline
\end{tabular}

Data are presented as median (IQR), $\mathrm{n}(\%)$, or mean \pm standard deviation, calculated from the mean per patient during ICU admission. Valve surgery includes mitral valve plasty, tricuspid valve plasty, aortic valve replacement or a combination of these. $C A B G$, Coronary artery bypass grafting; APACHE IV PM, Acute Physiology and Chronic Health Evaluation IV predicted mortality; SOFA, sequential organ failure assessment score per patient during the study period; EuroSCORE, European system for cardiac operative risk evaluation; $I C U$, intensive care unit; $R A D$, relative absolute deviation; $M F I$, microvascular flow index; $P P V$, proportion of perfused vessels; $P V D$, perfused vessel density; $\mathrm{SpO}_{2}$, peripheral oxygenation; $\mathrm{StO}_{2}$, tissue oxygenation; $H F$, heart frequency; $M A P$, mean arterial pressure.

was 4.0 (IQR, 2.0-5.0). The median APACHE IV PM and maximum Sequential Organ Failure score was 0.01 (IQR, 0.003-0.02) and 6.0 (IQR, 5.3-7.0), respectively. The patient characteristics are listed in Table 1.

\section{Microcirculation}

SDF recordings at 246 measurement points met our quality criteria for the MFI assessment, with a median of 4 points per patient (IQR, 3-5). For the PVD and PPV scoring, only 178 points were suitable because of excessive movement of 1 of the 3 recordings of that point (median, 3 per patient; IQR, 2-4). The median MFI, PVD, PPV, HI PVD, 
TABLE 2. Covariates associated with sensor accuracy

\begin{tabular}{|c|c|c|c|c|c|c|c|}
\hline \multirow[b]{2}{*}{ Variable } & \multicolumn{3}{|c|}{ Univariate } & \multicolumn{4}{|c|}{ Multivariate } \\
\hline & b & SE & $P$ value & b & SE & $P$ value & AIC \\
\hline Navigator & & & & & & & -1085.935 \\
\hline APACHE IV PM & 0.017 & 0.010 & $<.001$ & 0.017 & 0.004 & $<.001$ & \\
\hline Age & 0.002 & 0.001 & $<.001$ & 0.002 & 0.001 & .037 & \\
\hline Peripheral temperature & -0.009 & 0.003 & .001 & -0.008 & 0.003 & .003 & \\
\hline Dopamine dose & 0.008 & 0.003 & .006 & -0.001 & 0.004 & .785 & \\
\hline Enoximon dose & 0.016 & 0.006 & .008 & 0.009 & 0.007 & 207 & \\
\hline DM (no vs yes) & -0.038 & 0.016 & .019 & -0.024 & 0.018 & .177 & \\
\hline Ketanserine dose & 0.353 & 0.058 & $<.001$ & & & & \\
\hline Guardian & & & & & & & -758.090 \\
\hline Peripheral temperature & -0.006 & 0.003 & .036 & -0.006 & 0.003 & .048 & \\
\hline Dopamine dose & 0.007 & 0.003 & .046 & 0.004 & 0.004 & .336 & \\
\hline
\end{tabular}

Linear mixed-effects model for repeated measures, with the different patients as subjects and the 2-hour reference glucose measurements as repeated measures, using the autoregressive (1) repeated covariance structure. Sensor accuracy (ie, relative absolute deviation) was the dependent variable. The covariates were put into the model as fixed effects. AIC reflects model fit. Individual associations of variables with sensor accuracy (univariate) and basic model of both sensors including the variables with individual significant association with sensor accuracy (multivariate). SE, Standard error; AIC, Akaike's information criterion; APACHE IV PM, acute physiology and chronic health evaluation IV predicted mortality; $D M$, diabetes mellitus.

and $\mathrm{StO}_{2}$ was 2.8 (IQR, 2.7-2.9), 9.3 vessels $/ \mathrm{mm}$ (IQR, 8.4-10.0), 0.97 (IQR, 0.96-0.99), 0.17 (IQR, 0.13-0.21), and 90.3\% (IQR, 87.1\%-92.0\%), respectively (Table 1). The microcirculation variables were not different between patients with and without diabetes. The mean \pm standard deviation $\mathrm{StO}_{2}$ increased significantly during the first hours of ICU admission, from $88.6 \%$ (5.6) to a maximum of $91.2 \%$ (3.9) after 6 hours, and decreased thereafter, stabilizing after 20 hours (Figure 2). This increase in $\mathrm{StO}_{2}$ was accompanied by a decrease in PVD $(b=-0.697$, standard error, $0.267, P=.01$, linear mixed-effects model for repeated measures). In line with this observation, we found that the PVD was lowest in the first 8 hours after ICU admission compared with the next day (median, 9.2; IQR, 8.1-9.9; and median, 9.4; IQR, 8.6-10.5, respectively; $P=.049$, Wilcoxon signed ranks test). The MFI, PPV, and HI PVD did not change over time. The arterial oxygen content (hemoglobin concentration $\times$ pulse oximeter oxygen saturation $\times 1.34)$ was stable during ICU admission (mean \pm standard deviation, $12.6 \pm 1.3 \mathrm{~mL}$ oxygen $/ 100$ $\mathrm{mL}$ ). The MFI, PVD, and PPV were not influenced by administration of vasopressor medication (dopamine, norepinephrine, and enoximone). An increase in these medications did increase the $\mathrm{StO}_{2}$ on univariate analysis. However, the multivariate mixed-effects model analysis showed no significant relationship. Also, the crossclamp time during surgery was not related to the microcirculation variables.

\section{CGM Accuracy}

Clarke error grids of reference glucose values with the corresponding next sensor values are shown in Figure 1.
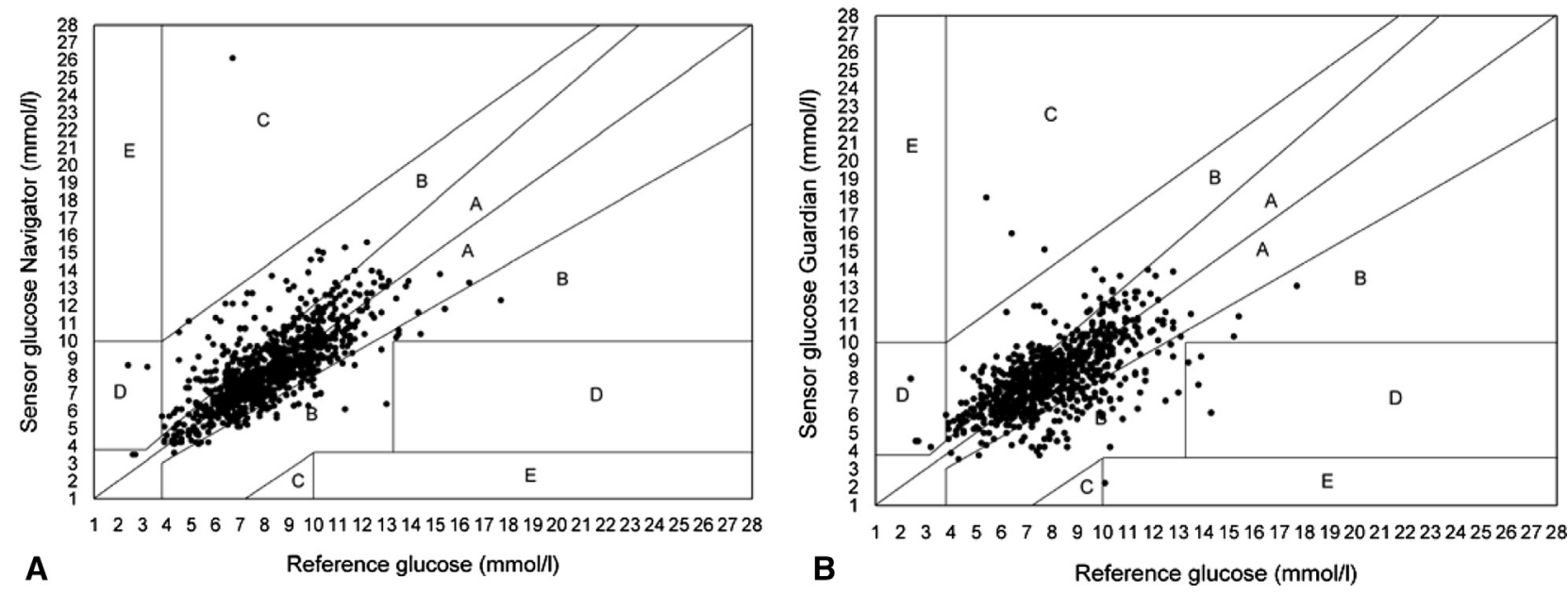

FIGURE 1. Clarke error grid of glucose measurements. A, Navigator, and B, Guardian. Each grid shows the data pairs of the reference glucose values at the $\mathrm{x}$-axis with proximate sensor values (within 10 minutes for the Navigator and 5 minutes for the Guardian) at the y-axis. 


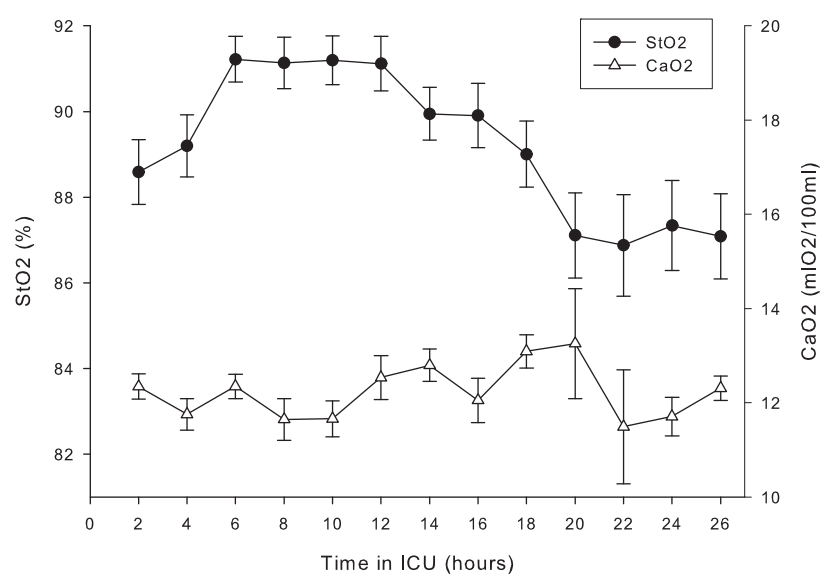

FIGURE 2. Tissue oxygenation $\left(\mathrm{StO}_{2}\right)$ in relation to arterial oxygen content $\left(\mathrm{CaO}_{2}\right)$ during intensive care unit (ICU) admission. Development of mean \pm standard error $\mathrm{StO}_{2}$ (left y-axis) and $\mathrm{CaO}_{2}$ (right y-axis) during ICU admission ( $\mathrm{x}$-axis).

For the comparisons with the arterial reference glucose values, $81.8 \%$ of the Navigator and $73.2 \%$ of the available Guardian glucose values were within zone A, and $17.7 \%$ of the Navigator and $25.2 \%$ of the Guardian glucose values were within zone B. Only 5 of the 934 Navigator values were within zone $\mathrm{C}$ or $\mathrm{D}(0.5 \%)$, with none in zone $\mathrm{E}$, compared with 13 of the 790 Guardian values in zone C, D, or E (1.3\%). Overall, the Navigator sensor performed significantly better than the Guardian sensor (median RAD, $11 \%$; IQR, 8-16; vs median, $14 \%$; IQR, 11-18; $P=.05){ }^{3}$

The variables that were individually associated with worse Navigator sensor accuracy were older age, a diagnosis of diabetes, a decreased peripheral temperature, an increase in the ketanserin, dopamine, or enoximone dose, and higher APACHE IV PM. Only a decreased peripheral temperature and increasing dopamine use were associated with decreased accuracy of the Guardian sensor (Table 2). None of the microcirculation variables (Table 3; an additional explanation of the microcirculation variables

TABLE 3. Forced introduction of microcirculatory variables

\begin{tabular}{lrcc}
\hline Variable & $\mathbf{B}$ & $\mathbf{S E}$ & $\boldsymbol{P}$ value \\
\hline Navigator & & & \\
MFIs & -0.021 & 0.041 & .61 \\
$\mathrm{PVD}$ & -0.003 & 0.007 & .63 \\
$\mathrm{PPV}$ & 0.147 & 0.307 & .63 \\
$\mathrm{StO}_{2}$ & 0.002 & 0.001 & .12 \\
Guardian & & & \\
$\mathrm{MFIs}$ & -0.101 & 0.063 & .11 \\
$\mathrm{PVD}$ & 0.009 & 0.009 & .33 \\
$\mathrm{PPV}_{\mathrm{StO}}$ & -0.256 & 0.448 & .57 \\
\hline
\end{tabular}

The forced introduction of the microcirculatory variables in turn did not show a significant association with sensor accuracy. SE, Standard error; $M F I s$, microvascular flow index of small vessels; $P V D$, perfused vascular density; $P P V$, proportion of perfused vessels; $\mathrm{StO}_{2}$, tissue oxygen saturation. is provided below) nor gender, mean arterial pressure, norepinephrine dose, or nitroglycerine dose showed significant associations with the accuracy of either of the sensors.

Subsequently, we built a multivariate model per sensor that included the significantly associated factors (Table 2). Owing to collinearity between the APACHE IV PM and the ketanserin dose, we only included APACHE IV PM in the final model. This model showed that for the Navigator only a higher APACHE IV PM, lower peripheral temperature, and older age remained significantly associated with an increase in sensor RAD (ie, worse sensor accuracy). For the Guardian, a decrease in the peripheral temperature remained associated with an increase in the RAD. Figure 3 shows the crude accuracy of both sensors stratified by the peripheral temperature. Successively forcing these variables in turn into the model did not reveal a significant association with sensor accuracy (Table 3). It was not possible to completely eliminate the glucose values in the $\mathrm{C}, \mathrm{D}$, and $\mathrm{E}$ zones using various cutoff values for temperature, age, and APACHE IV PM score.

\section{DISCUSSION}

In the present study, we found acceptable continuous glucose sensor accuracy and microcirculatory impairment to a limited extent in cardiac surgery patients in the first hours of ICU admission after surgery during a median follow-up of 23 hours. Microcirculatory impairment was reflected by a decrease in PVD and a transient increase in $\mathrm{StO}_{2}$ but no differences in PPV and MFI. The microcirculatory variables showed no relationship with continuous glucose sensor accuracy. The variables associated with worse sensor accuracy were older age, lower peripheral temperature, and higher APACHE IV PM for the Navigator

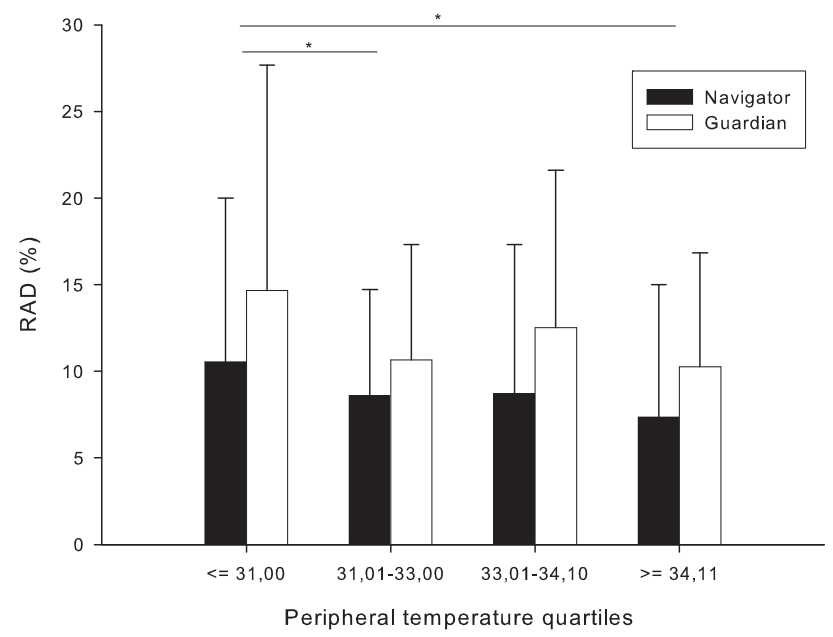

FIGURE 3. Sensor accuracy in relation to peripheral temperature. Median (interquartile range) relative absolute deviation ( $R A D)$ of both sensors stratified by peripheral temperature quartile. The highest RAD was seen at $<31^{\circ} \mathrm{C}$ and the lowest $\mathrm{RAD}$ at $>34.11^{\circ} \mathrm{C}$. $* P<.05$. 
sensor and lower peripheral temperature for the Guardian sensor.

Our results of the accuracy of the Guardian sensor are comparable to those from Logtenberg et $\mathrm{al}^{5}{ }^{5}$ who also performed a study of cardiac patients in the ICU and reported a median RAD of $12.3 \%$ during the ICU period. No studies using the Navigator in the ICU have been published to date. Compared with the data from patients with type 1 diabetes, the accuracy of both devices in our ICU population was comparable for the Guardian ${ }^{18}$ and even somewhat better for the Navigator. ${ }^{19}$ This suggests that at least in the population of cardiac surgery patients, CGM use is feasible.

A decrease in the peripheral temperature did influence the accuracy of both sensors negatively. This finding was in contrast to that of 1 other study performed in pediatric cardiac surgery patients using the older Guardian RT CGM system, which did not show a relationship. ${ }^{20}$ It is, however, plausible that the temperature of the skin influences the sensor accuracy, because the optimal reaction temperature for the glucose oxidase enzyme incorporated in the sensors is $30^{\circ} \mathrm{C}$ to $40^{\circ} \mathrm{C}$, and the skin temperature during and shortly after cardiac surgery is often less than that lower limit. Nevertheless, the decreased sensor accuracy needs to be put into clinical perspective, because the median and IQR RAD of both sensors during peripheral temperatures $<31{ }^{\circ} \mathrm{C}$ is still relatively low (Navigator, $10.5 \%$, 4.8\%-20.0\%; Guardian, $14.7 \%$, 6.6\%-27.7\%; Figure 2).

The performance of the Navigator was also negatively influenced by older age and higher APACHE IV PM. As we hypothesized, the highest RAD was found in the more severely ill patients because of more out-of-range glucose values; however, apparently the microcirculatory variables used did not mediate this effect in those with the highest APACHE IV PM. The APACHE IV PM in our patient group was relatively low; therefore, these results should not be extrapolated to patients with higher severity of disease scores. Age contributes to the APACHE IV PM and also might be associated with skin-related factors influencing sensor performance (eg, altered subcutaneous fat deposition or vascularization). Notably, the accuracy of the Navigator was influenced by more variables than was the accuracy of the Guardian. Possibly, the inaccuracy of the Guardian was largely dependent on sensor-related factors overruling the influence of the patient-related variables.

Previous studies of microcirculatory function in postoperative cardiac surgery patients have shown varying results. Some have found normal MFI values at ICU arrival $^{21}$ and normal PVD values 1 hour after cardiopulmonary bypass. ${ }^{22}$ In contrast, De Backer et $\mathrm{al}^{23}$ found a decrease in PPV at the end of on-pump surgery that persisted until 24 hours after surgery. Of note, other than the present study, the latter study is the only study with
$>1$ hour of follow-up during postoperative ICU admission of cardiac surgery patients. The MFI, PPV, and HI found in our population were roughly comparable to those of healthy controls, as measured by Trzeciak et al. ${ }^{15}$ The PVD in our group was somewhat smaller than in their control group and not as low as in patients with sepsis. ${ }^{7}$ However, we did not have the opportunity to measure the microcirculatory function preoperatively; therefore, we were unable to assess the pre- to postoperative changes.

We found an increase in $\mathrm{StO}_{2}$ after ICU admission, with a peak after 6 to 12 hours (Figure 2) and a gradual decrease thereafter, stabilizing to normal levels $<90 \%$ after 20 hours of ICU stay. No studies measuring $\mathrm{StO}_{2}$ in patients after cardiac surgery are known to us. Together with the increase in $\mathrm{StO}_{2}$, the lowest PVD values were found in the first 8 hours of ICU admission, and the increase in $\mathrm{StO}_{2}$ was significantly associated with the decrease in PVD. Endotoxin release and subsequent triggering of the inflammatory response observed during on-pump surgery could explain these findings. Endotoxin levels peak shortly after surgery, and the inflammatory response, represented by the plasma interleukin- 6 concentration, is found to be highest after 6 hours, ${ }^{24}$ comparable to the observed peak in $\mathrm{StO}_{2}$. A much larger inflammatory response will be seen with sepsis and is accompanied by large microcirculatory changes ${ }^{7}$ and also a possible increase in $\mathrm{StO}_{2}$ owing to impaired oxygen offloading in a hyperdynamic flow state. ${ }^{25}$ The changes seen in our population could thus have been due to changes similar to, but much smaller than, those seen in sepsis caused by distributive shock, owing to arteriovenous shunting of the microcirculation reducing the number of perfused vessels and thereby disabling oxygen offloading. This hypothesis is supported by the stable arterial oxygen content during ICU admission (Figure 2), which makes it unlikely that the increased $\mathrm{StO}_{2}$ could be explained by an increase in the oxygen supply. One limitation of the present study was that we were not able to investigate the relation between the microcirculation parameters and the cardiac output as a measure of the hemodynamic situation. This was because in our unit almost no patients will have Swan-Ganz catheters inserted, and, therefore, measurement of the cardiac output is seldom available.

The microcirculatory variables did not influence the accuracy of either sensor. As far as we know, no previous studies have assessed this relationship. Our findings suggest that the microcirculatory status does not impair glucose transport in cardiac surgery patients, at least not to the extent that it has an effect on the subcutaneous sensor performance. The patients included in the present study had a relatively good microcirculation as measured using the MFI, PPV, and PVD, although the differences between patients were quite large (PVD ranged from 5.9 to 13.8 vessels $/ \mathrm{mm}$ ). It remains to be established whether in 
patients with worse overall microcirculatory variables, the sensor performance will be affected.

In conclusion, the present study has shown that the sensor accuracy of both sensors was acceptable in cardiac surgery patients. The microcirculation showed some impairment after surgery, reflected by a transient increase in $\mathrm{StO}_{2}$ and a decrease in PVD; however, overall, it was quite good compared with that in patients with sepsis and healthy controls. The impairment in the microcirculatory variables was not related to sensor accuracy but the peripheral temperature (both sensors) and age and APACHE IV PM (Navigator) was. These results support CGM use in cardiac surgery patients characterized by a low severity of illness. Additional studies are needed to assess the influence of microcirculatory changes on sensor accuracy in more severely ill patients.

The sensors used were provided free of charge by Medtronic Minimed and at a discounted rate by Abbott Diabetes Care.

\section{References}

1. Stamou SC, Nussbaum M, Carew JD, Dunn K, Skipper E, Robicsek F, et al. Hypoglycemia with intensive insulin therapy after cardiac surgery: predisposing factors and association with mortality. J Thorac Cardiovasc Surg. 2011;142:166-73.

2. Holzinger U, Warszawska J, Kitzberger R, Wewalka M, Miehsler W, Herkner H, et al. Real time continuous glucose monitoring in critically ill patients-a prospective, randomized trial. Diabetes Care. 2010;33:467-72.

3. Siegelaar SE, Barwari T, Hermanides J, Stooker W, van der Voort PHJ, Devries JH. Accuracy and reliability of continuous glucose monitoring at the ICU: a head to head comparison of two subcutaneous glucose sensors in cardiac surgery patients. Diabetes Care. 2011;34:e31.

4. Furnary AP, Gao G, Grunkemeier GL, Wu Y, Zerr KJ, Bookin SO, et al. Continuous insulin infusion reduces mortality in patients with diabetes undergoing coronary artery bypass grafting. J Thorac Cardiovasc Surg. 2003;125:1007-21.

5. Logtenberg SJ, Kleefstra N, Snellen FT, Groenier KH, Slingerland RJ, Nierich AP, et al. Pre- and postoperative accuracy and safety of a real-time continuous glucose monitoring system in cardiac surgical patients: a randomized pilot study. Diabetes Technol Ther. 2009;11:31-7.

6. Rabiee A, Andreasik RN, Abu-Hamdah R, Galiatsatos BS, Khouri Z, Gibson BR, et al. Numerical and clinical accuracy of a continuous glucose monitoring system during intravenous insulin therapy in the surgical and burn intensive care units. J Diabetes Sci Technol. 2009;3:951-9.

7. De Backer D, Creteur J, Preiser JC, Dubois MJ, Vincent JL. Microvascular blood flow is altered in patients with sepsis. Am J Respir Crit Care Med. 2002;166:98-104.

8. De Backer D, Creteur J, Dubois MJ, Sakr Y, Vincent JL. Microvascular alterations in patients with acute severe heart failure and cardiogenic shock. Am Heart J. 2004;147:91-9.
9. Rood E, Bosman RJ, van der Spoel JI, Taylor P, Zandstra DF. Use of a computerized guideline for glucose regulation in the intensive care unit improved both guideline adherence and glucose regulation. J Am Med Inform Assoc. 2005; 12:172-80.

10. Goedhart PT, Khalilzada M, Bezemer R, Merza J, Ince C. Sidestream dark field (SDF) imaging: a novel stroboscopic LED ring-based imaging modality for clinical assessment of the microcirculation. Opt Express. 2007;15:15101-14.

11. Verdant CL, De Backer D, Bruhn A, Clausi CM, Su F, Wang Z, et al. Evaluation of sublingual and gut mucosal microcirculation in sepsis: a quantitative analysis. Crit Care Med. 2009;37:2875-81.

12. Elbers PW, Ozdemir A, Heijmen RH, Heeren J, van Iterson M, van Dongen EP, et al. Microvascular hemodynamics in human hypothermic circulatory arrest and selective antegrade cerebral perfusion. Crit Care Med. 2010;38:1548-53.

13. De Backer D, Hollenberg S, Boerma C, Goedhart P, Buchele G, OspinaTascon G, et al. How to evaluate the microcirculation: report of a round table conference. Crit Care. 2007;11:R101.

14. Boerma EC, Mathura KR, van der Voort PH, Spronk PE, Ince C. Quantifying bedside-derived imaging of microcirculatory abnormalities in septic patients: a prospective validation study. Crit Care. 2005;9:R601-6.

15. Trzeciak S, Dellinger RP, Parrillo JE, Guglielmi M, Bajaj J, Abate NL, et al. Early microcirculatory perfusion derangements in patients with severe sepsis and septic shock: relationship to hemodynamics, oxygen transport, and survival. Ann Emerg Med. 2007;49:88-98.

16. Cohn SM, Nathens AB, Moore FA, Rhee P, Puyana JC, Moore EE, et al Tissue oxygen saturation predicts the development of organ dysfunction during traumatic shock resuscitation. J Trauma. 2007;62:44-54.

17. Clarke WL, Cox D, Gonder-Frederick LA, Carter W, Pohl SL. Evaluating clinical accuracy of systems for self-monitoring of blood glucose. Diabetes Care. 1987; $10: 622-8$

18. Mazze RS, Strock E, Borgman S, Wesley D, Stout P, Racchini J. Evaluating the accuracy, reliability, and clinical applicability of continuous glucose monitoring (CGM): is CGM ready for real time? Diabetes Technol Ther. 2009;11:11-8.

19. Weinstein RL, Schwartz SL, Brazg RL, Bugler JR, Peyser TA, McGarraugh GV. Accuracy of the 5-day FreeStyle Navigator continuous glucose monitoring system: comparison with frequent laboratory reference measurements. Diabetes Care. 2007;30:1125-30.

20. Piper HG, Alexander JL, Shukla A, Pigula F, Costello JM, Laussen PC, et al. Real-time continuous glucose monitoring in pediatric patients during and after cardiac surgery. Pediatrics. 2006;118:1176-84.

21. den Uil CA, Lagrand WK, Spronk PE, van Domburg RT, Hofland J, Luthen C et al. Impaired sublingual microvascular perfusion during surgery with cardiopulmonary bypass: a pilot study. J Thorac Cardiovasc Surg. 2008;136 129-34.

22. Bauer A, Kofler S, Thiel M, Eifert S, Christ F. Monitoring of the sublingual microcirculation in cardiac surgery using orthogonal polarization spectral imaging: preliminary results. Anesthesiology. 2007;107:939-45.

23. De Backer D, Dubois MJ, Schmartz D, Koch M, Ducart A, Barvais L, et al. Microcirculatory alterations in cardiac surgery: effects of cardiopulmonary bypass and anesthesia. Ann Thorac Surg. 2009;88:1396-403.

24. Boelke E, Storck M, Buttenschoen K, Berger D, Hannekum A. Endotoxemia and mediator release during cardiac surgery. Angiology. 2000;51:743-9.

25. Elbers PW, Ince C. Mechanisms of critical illness-classifying microcirculatory flow abnormalities in distributive shock. Crit Care. 2006;10:221. 